\title{
A Robotic Path Planning by Using Crow Swarm Optimization Algorithm
}

\author{
Mohammed Yousif \\ Directorate of Regions and Governorates Affairs, Ministry of Youth \& Sport, Iraq. \\ E-mail: mohammed.alyousif1991@gmail.com
}

\author{
Ahmad Salim \\ Middle Technical University, Baghdad, Iraq \\ E-mail: ahmadsalim@mtu.edu.iq,pro.ahmadsalimiq@gmail.com
}

\author{
Wisam K. Jummar \\ University of Anbar, Anbar, Iraq \\ E-mail: wisamkhalid6@uoanbar.edu.iq
}

Received: 28 December 2020; Accepted: 18 January 2021; Published: 08 February 2021

\begin{abstract}
One of the most common problem in the design of robotic technology is the path planning. The challenge is choosing the robotics' path from source to destination with minimum cost. Meta-heuristic algorithms are popular tools used in a search process to get optimal solution. In this paper, we used Crow Swarm Optimization (CSO) to overcome the problem of choosing the optimal path without collision. The results of CSO compared with two meta-heuristic algorithms: PSO and ACO in addition to a hybrid method between these algorithms. The comparison process illustrates that the CSO better than PSO and ACO in path planning, but compared to hybrid method CSO was better whenever the smallest population. Consequently, the importance of research lies in finding a new method to use a new metahumanistic algorithm to solve the problem of robotic path planning.
\end{abstract}

Index Terms: Robotic, Path planning, Meta-heuristic, Crow Swarm Optimization (CSO), Optimization.

\section{Introduction}

Nowadays, robotic takes many human's roles in many fields such as military, agriculture and industry [1]. Due to the rapid growth in technology, robots are getting done the human tasks where the robots have proven to be better in terms of speed and accuracy [2][3]. One of the biggest problem in the robotic technology is the process of move from a source to destination without collision and with minimum cost in terms of distance and time [4], it is called robotic path planning or plano mover's problem. The robotics world is a hybrid between artificial intelligence and mechanical science [5]. Robots are now able to make decisions on their own and without the intervention of the human factor, as they can move, monitor and make decisions in many vital areas that facilitate human life [6]. In the robotic technology, there are two types of environments: the local and the global environment.

In a local environment, the obstacles' position changes permanently so the robotic must be chosen their next movement carefully in order to avoid collision. In contrast, the positions of obstacles static and never change in global environment, that means the robotics' path from the source to the destination known previously. Recently, metaheuristic optimization algorithms popularly is used to solve many problem instead of heuristic techniques to overcome the local minimum problem. Meta-heuristic algorithms not greedy in nature and balance between local and global of the optimal solution for these reasons they utilize to solve robotic path planning.

There are many researches employed to address the robotic path planning problem those used meta-heuristic algorithms. The main objective of this paper is to use CSO in solving the robotic path planning problem and measuring the efficiency of the algorithm in solving problems of this type. The main contribution of this research is the use of CSO [7] algorithm to solve robotic path planning. Moreover, comparing the results of many meta-heuristic algorithms to know the behaver of these algorithms in solving this problem. The CSO was more efficient in finding the robotic path in the smaller the population.

The remain of this paper arranged as follows: in section 2 shown the most related works, section 3 illustrates CSO algorithm, section 4 explain the suggested method to overcome the search problem, section 5 discusses the results of paper and section 6 concludes the paper. 


\section{Related Work}

Today, meta-heuristic optimizers utilize to solve many kinds of problem, one of them is the robotic path planning. There are many researchers used meta-heuristic algorithms to minimize the cost of robotics' path and to find the free obstacles path.

Gigras et. al. in [8], proposed a very interested hybrid method that has been used to overcome robotic path planning problem by merge between two common meta-heuristic algorithms, these are: Ant Colony Optimization (ACO) [9] and Particle Swarm Optimization (PSO) [10], the suggested method get a very good results compared with each algorithm alone but it is very complex method. Chen in [11], proposed an improvement on ACO to make it suitable to the robotic path planning problem, the main idea to change ACO id to split the algorithm into two steps: preprocessing and path planning, the suggested changes obtained good performance to find best path for robot.

Salawudeen et. al. in [12], proposed an new method for robotic path planning. The proposed method used three meta-heuristic algorithms: Smell Agent Optimization (SAO), PSO and Smell Detection Agent (SDA) to explore the robotic path with least cost and free obstacle. The result shows that the SAO gets a better fitness than other algorithms. In addition to that, there are many researchers who have used Meta-heuristic algorithms hybrid with other methods to solve the path planning problem, such as using whale optimization algorithm (WOA) with fuzzy [13].

Dao et. al. in , presented a robotic path planning method that depends on modified Whale optimization algorithm (WOA) [14] called multi-objective whale optimization algorithm (MWOA, the proposed technique used the obstacles and target point to calculate a fitness of WOA and provide good results. All the aforementioned works used metaheuristic algorithms to solve the robotic path planning problem, but the results were different between each work and the other as each search had improvement in a specific area and failure in another area within the robotic search environment.

\section{Crow Swarm Optimization (CSO)}

CSO is a meta-heuristic optimization algorithm inspired from the behavior of American Crow, processed by Yousif [7]. Initially, CSO proposed to overcome Traveling Salesman Problem (TSP) but in this work that uses to process the robotic planning problem in addtion to time table problem and robotic path problem. The idea of CSO is that each crow randomly searches in the problem space to find the optimal space or target by adjusted its parametrs based on its own memory and the information collected from other crows.

There are many parameters must be initialize before start search with CSO, these parameters are: number of crows, number of groups and number of iterations. First of all, the population initializes randomly, then the fitness of solutions in the population must be calculate. Equation 1 represents the inspired of crow's behavior.

$$
S=\left(R * S_{i}\right)+\left(P_{b e s t}+P_{i}\right) * R
$$

where $\mathrm{i}$ is a counter indicates the current iteration, $\mathrm{R}$ is random value between $(0,1)$, Pbest is the position vector of the best solution obtained so far, $\mathrm{Pi}$ is the position vector and $\mathrm{Si}$ is the velocity value, it is between $(-6,6)$. The solution must be updated during every iteration, the updating of solutions aims to improve the worst solutions by conveying the effect of the best solutions. Equation 2 is used to update and to improve the solutions, where $\mathrm{P}$ is represented the position of crow and $\Theta$ represented the crow's angle. The angle of worst solution changes based on equation 3.

$$
\begin{gathered}
P_{i+1}=P_{i}+S_{i+1}+\cos (\theta) \\
\Theta_{i+1}=\frac{\theta_{i}+\theta_{\text {best }}}{2}
\end{gathered}
$$

\section{Methodology}

Path planning is used to choose the optimal path from source to target point in search space without collision and with a minimum cost. Robot uses a path planning to find the best path with avoiding collision with any of the obstacles, when robot collided it must be stopped and take some steps back. In this work to achieve this search process CSO metaheuristic algorithm is used. The difficulty of the robotic path planning problem is finding the least cost path without colliding with obstacles. Figure 1 shown an example of robotic path planning search space.

CSO is a meta-heuristic searching algorithm, to use it in robotic path planning problem especial list used to save the path from source to destination that is called path-list. Initially, path-list contain one point only before fist iteration start it is a source point. Circularly, each iteration the best solution adds to the list of points. Algorithm 1 shown the function that calculates the fitness of population solutions, where the optimality of solutions depends on the distance of the candidate point and the target point, provided that it does not collide with any of the obstacles within the search 
space. Best solution (point) it is most nearest point to target point (that is get less distance from fitness function), Manhattan distance [15] in equation 4 explains how to calculate the distance between candidate and target point. Moreover, each iteration the best solution (it is a solution with lest fitness value in the population) uses to optimize the worst solutions rely on equation 1,2 and 3 .

$$
M D\left(P_{1}, P_{2}\right)=\left|x_{1}-x_{2}\right|+\left|y_{1}-y_{2}\right|
$$

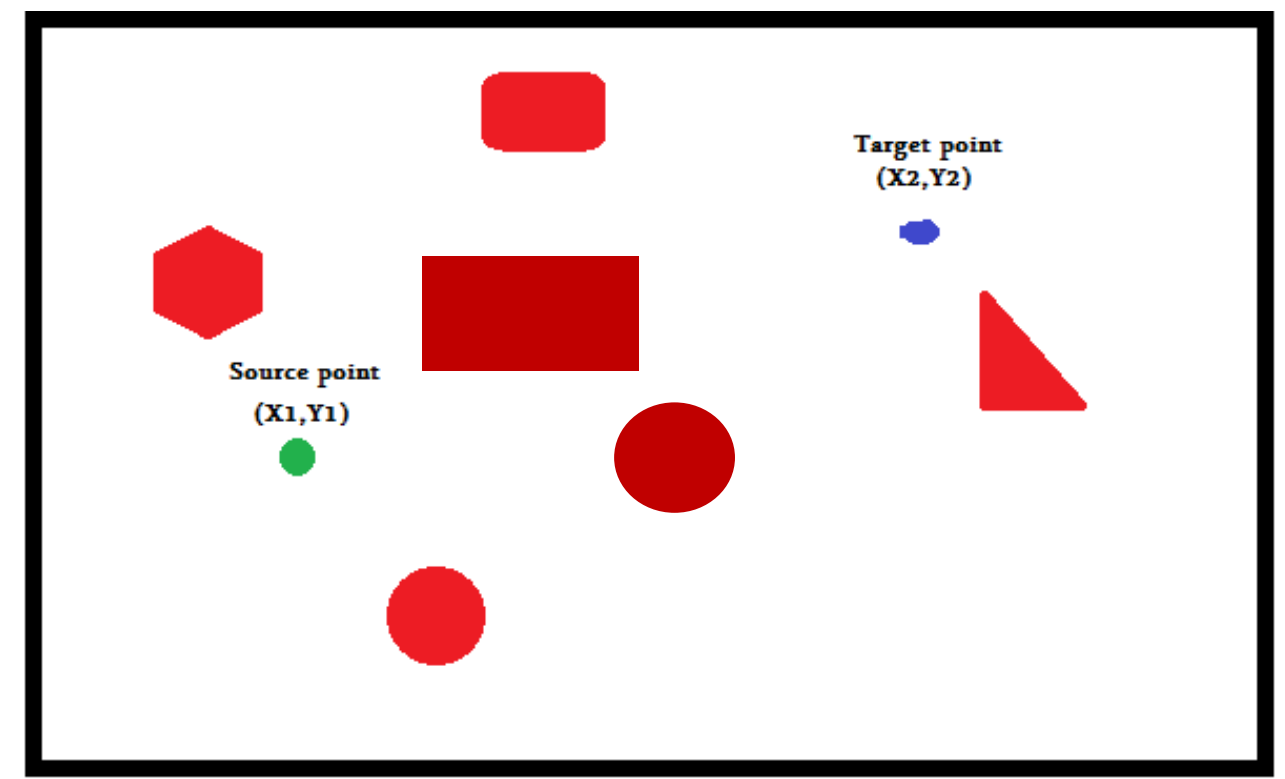

Fig.1. Example of Robot's search space.

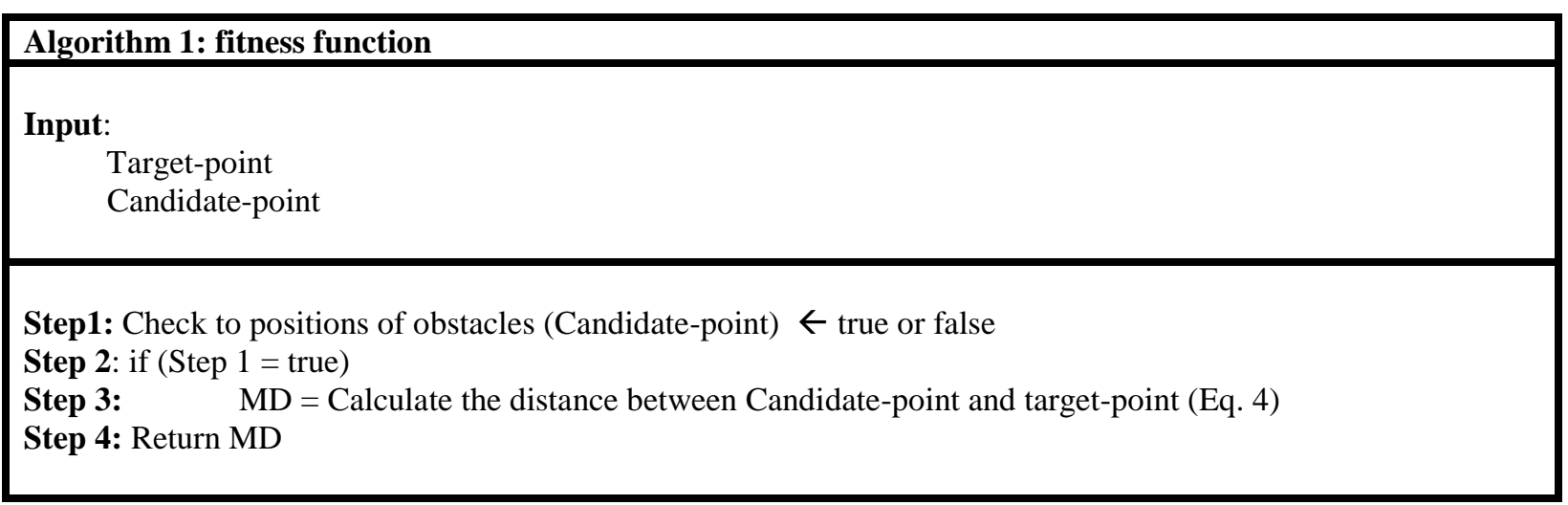

Algorithm 2 illustrates the complete search process from source to destination point. The proposed method to find robot's path do not need a mechanism to return from collision because it avoids collision before moving by knowing the position of the obstacles before each movement, and if the two bodies move at the same time to the same point, the robot back to the last point in which they do not collide with any object.

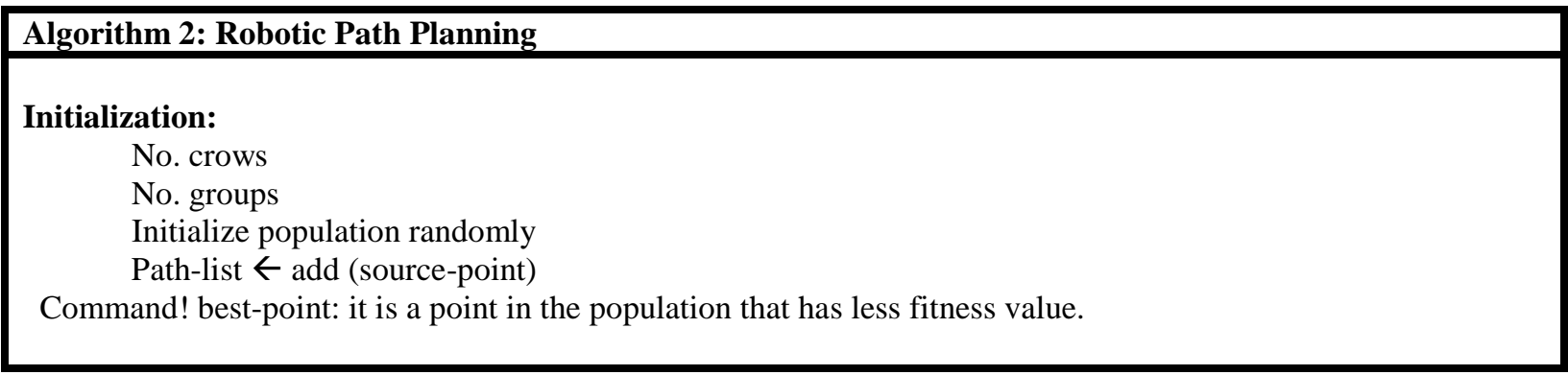


Step 1: Calculate fitness function (population (algorithm 1))

Step 2: Loop

Step 3: $\quad$ if (not collided (best-point) and (target-point $\neq$ best-point))

Step 4: $\quad$ add to path-list(best-point)

Step 5: $\quad$ back to last not collided point in the path-list

Step 6: $\quad$ if (target-point $=$ best-point) $)$

Step 7: add to path-list(best-point)

Step 8: $\quad$ stop Loop

Step 9: $\quad$ Optimize solutions (population) (Eq.1, Eq.2 and Eq.3)

Step 10: Calculate fitness function (population)

End loop

Step 11: Return path-list

\section{Results and Discussion}

In this section, the proposed robotic path planning technique is investigated. The robotic path planning through the simulation of CSO gave impressive results indicating the importance of applying this approach, which outperforms other methods. As shown in Table 1, the number of repetitions is at a minimum through the application of this approach, through which the optimal path of movement is also drawn. Likewise, while moving in the path that is chosen to move from the starting point until reaching the end point, the existing obstacles are avoided and collision with them is prevented.

Table 1. Comparison between CSO, PCO and ACO.

\begin{tabular}{|c|c|c|c|c|}
\hline \multirow{2}{*}{ No_population } & \multicolumn{4}{|c|}{ Algorithms and number of iterations } \\
\cline { 2 - 5 } & PSO & ACO & ACO\&PSO & CSO \\
\hline $\mathbf{1 0}$ & 2025 & 480 & 160 & 115 \\
\hline $\mathbf{2 0}$ & 2070 & 395 & 160 & 143 \\
\hline $\mathbf{3 0}$ & 2100 & 316 & 160 & 157 \\
\hline $\mathbf{4 0}$ & 2310 & 316 & 158 & 177 \\
\hline $\mathbf{5 0}$ & 2400 & 316 & 158 & 194 \\
\hline
\end{tabular}

As we note from the above table, the results obtained from the proposed technique are better than PSO and ACO, but in the case of comparison with ACO\&PSO, we see the superiority of the proposed algorithm also, but when the population increases, this algorithm will give slightly better results than the proposed algorithm.

This algorithm can be greatly utilized if applied to moving obstacles. Thus, it will turn into a fully dynamic environment. This is not an easy topic, but it can be worked on in the future. Robots' movement paths can be drawn through a fair number of areas of application, and these paths are very useful for human life in various fields, the most important of which is medical science. Recently, several hospitals have used robotic devices in their surgeries, with good success. Finally, the use of CSO in solving the robotic path planning problem gave competitive results compared to the rest of the algorithms and was much better than other algorithms separately, but it was better compared to the hybrid methods in case the population was small only.

\section{Conclusion}

This work used CSO meta-heuristic algorithm to present a new robotic path planning technique. The results analysis of proposed technique showed that it has a good performance and CSO algorithm can effectively find the optimal robot's path with minimum cost and without collision with any obstacles.

CSO was better than other meta-heuristic algorithm such as PSO and ACO used individually to solve the robotic path planning problem and comparison to hybrid techniques the suggest method was very comparative especially when the population size was small. Therefore, we expect the combination of CSO and other meta-heuristic algorithms may be given very excellent results. In the end, the contribution of the research in employing CSO lies in solving the robotic path planning problem, and the results of this proposed method were good compared to the rest of the methods used to solve this problem. 


\section{References}

[1] M. Mariappan, T. W. Fang, M. Nadarajan, and N. Parimon, "Face Detection and Auto Positioning for Robotic Vision System.," International Journal of Image, Graphics \& Signal Processing, vol. 7, no. 12, 2015.

[2] A. K. Tiwari and S. V. Nadimpalli, "New Fusion Algorithm provides an alternative approach to Robotic Path planning," arXiv preprint arXiv:2006.05241, 2020.

[3] A. Agrawal and M. P. Brijpuria, "A Dynamic Object Identification Protocol for Intelligent Robotic Systems," IJ Image, Graphics and Signal Processing, vol. 8, pp. 35-41, 2015.

[4] M. A. H. Eljinini and A. Tayyar, "Collision-free Random Paths between Two Points," International Journal of Intelligent Systems and Applications, vol. 12, no. 3, p. 27, 2020.

[5] R. Malik and S. Prasad, "Robot navigation and exploration in an unknown environment," in Robotic Systems, Springer, 1992, pp. 423-430.

[6] P. Pandey, A. Shukla, and R. Tiwari, "Aerial path planning using meta-heuristics: a survey," in 2017 second international conference on electrical, computer and communication technologies (ICECCT), 2017, pp. 1-7.

[7] M. Yousif and B. Al-Khateeb, "A Novel Metaheuristic Algorithm for Multiple Traveling Salesman Problem," Adv. Res. Dyn. Control Syst, vol. 10, no. 13, pp. 2113-2122, 2018.

[8] Y. Gigras, K. Choudhary, K. Gupta, and others, "A hybrid ACO-PSO technique for path planning," in 2015 2nd International Conference on Computing for Sustainable Global Development (INDIACom), 2015, pp. 1616-1621.

[9] M. Dorigo, M. Birattari, and T. Stutzle, "Ant colony optimization," IEEE computational intelligence magazine, vol. 1, no. 4, pp. 28-39, 2006.

[10] J. Kennedy and R. Eberhart, "Particle swarm optimization," in Proceedings of ICNN'95-International Conference on Neural Networks, 1995, vol. 4, pp. 1942-1948.

[11] X. Chen, Y. Kong, X. Fang, and Q. Wu, "A fast two-stage ACO algorithm for robotic path planning," Neural Computing and Applications, vol. 22, no. 2, pp. 313-319, 2013.

[12] A. T. Salawudeen et al., "Recent Metaheuristics Analysis of Path Planning Optimaztion Problems," in 2020 International Conference in Mathematics, Computer Engineering and Computer Science (ICMCECS), 2020, pp. 1-7.

[13] S. Raiesdana, “A Hybrid Method for Industrial Robot Navigation,” Journal of Optimization in Industrial Engineering, vol. 14, no. 1, pp. 219-234, 2021.

[14] S. Mirjalili and A. Lewis, "The whale optimization algorithm," Advances in engineering software, vol. 95, pp. 51-67, 2016.

[15] A. Vadivel, A. K. Majumdar, and S. Sural, "Performance comparison of distance metrics in content-based image retrieval applications," in International Conference on Information Technology (CIT), Bhubaneswar, India, 2003, pp. $159-164$.

\section{Authors' Profiles}

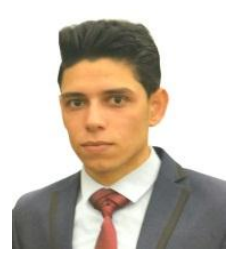

Mohammed Yousif was born in Anbar, Iraq in 1991. He received the B.S from Department of Computer Science, Computer Science and Information Technology, University of Anbar, Anbar, Iraq in 2016. He received Master at College of Computer Science and Information Technology, University of Anbar, Anbar, Iraq in 2019. His research interests now artificial intelligent include: Meta-heuristic, Robotics, Deep Learning.

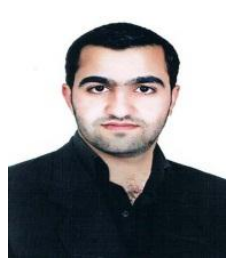

Ahmad Salim is an Assistant Lecture in Middle Technical University. He received his B.Sc. in Computer Science (2011) and M.Sc. in Computer Science (2018) from the University of Anbar, Iraq. He is interested in the following fields; Biometric, Information Security, Data Mining, Image Processing and Artificial Intelligence. He has published many papers in different scientific journals.

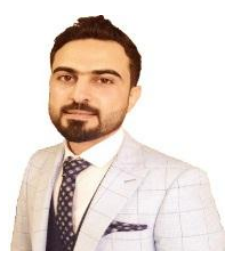

Wisam K. Jummar was born in Anbar, Iraq in 1986. He received the B.Sc. in (2009) and the master degree in 2019 from the faculty of the Computer Sciences and Information Technology, University of Anbar, Iraq. He is one of the faculty members within the presidency of Anbar University. His research interest includes image processing, data security, and data mining. 
How to cite this paper: Mohammed Yousif, Ahmad Salim, Wisam K. Jummar," A Robotic Path Planning by Using Crow Swarm Optimization Algorithm ", International Journal of Mathematical Sciences and Computing(IJMSC), Vol.7, No.1, pp. 20-25, 2021. DOI: $10.5815 /$ ijmsc.2021.01.03 